\title{
Image Inpainting Considering Brightness Change and Spatial Locality of Textures and Its Evaluation
}

\author{
Norihiko Kawai, Tomokazu Sato, and Naokazu Yokoya \\ Graduate School of Information Science, Nara Institute of Science and Technology \\ 8916-5 Takayama, Ikoma, Nara 630-0192, Japan \\ \{norihi-k, tomoka-s, yokoya\} @is.naist.jp \\ http://yokoya.naist.jp/
}

\begin{abstract}
Image inpainting techniques have been widely investigated to remove undesired objects in an image. Conventionally, missing parts in an image are completed by optimizing the objective function using pattern similarity. However, unnatural textures are easily generated due to two factors: (1) available samples in the image are quite limited, and (2) pattern similarity is one of the required conditions but is not sufficient for reproducing natural textures. In this paper, we propose a new energy function based on the pattern similarity considering brightness changes of sample textures (for (1)) and introducing spatial locality as an additional constraint (for (2)). The effectiveness of the proposed method is successfully demonstrated by qualitative and quantitative evaluation. Furthermore, the evaluation methods used in much inpainting research are discussed.
\end{abstract}

Keywords: Image Inpainting, Energy Minimization, Evaluation Method.

\section{Introduction}

Image inpainting methods have been widely investigated to remove undesired visual objects in an image. These methods can be classified into two categories. One is a non-exemplar-based method and the other is an exemplar-based method. The non-exemplar-based methods [1 234 56 6/891011] are based on pixel interpolation considering the continuity of pixel intensity. These methods are effective for small image gaps like scratches in a photograph. However, the resultant image easily becomes unclear when the missing region is large. Therefore, recently many exemplar-based inpainting methods have been intensively developed because they can synthesize complex textures in the missing region.

Exemplar-based methods basically synthesize textures for the missing region based on pattern similarity that is defined between the missing region and the rest of the image (data region). Some of the exemplar-based methods use the distance in the feature space as a similarity measure. As the feature space, Fourier space, wavelet domain and eigenspace have been used [121314]. Most of the other exemplar-based methods simply employ SSD (Sum of Squared Differences)-based pattern similarity measures [15/16 17/18 19 20|21 22 23 24]. Efros et al. [15] have proposed a method that successively copies the most similar

T. Wada, F. Huang, and S. Lin (Eds.): PSIVT 2009, LNCS 5414, pp. 271 282, 2009.

(C) Springer-Verlag Berlin Heidelberg 2009 
pattern from the data region to the missing region. Although this method can generate complex textures, the quality of resultant images is severely affected by the order of texture copy. To obtain good results with successive texture copy, confidence maps such as the number of fixed pixels in a window, strength of isophotes around the missing regions and pattern similarity have been used to determine the order of texture copy [1617/8]. Although the duplication of similar textures preserves the local texture continuity in these methods, discontinuous textures are easily synthesized in the completed image. To avoid the ordering problem, recent inpainting methods employ the iterative global optimization approach 192021. In these methods, the objective functions that evaluate the pattern similarity are defined and optimized by using EM algorithm, Belief Propagation approach and graph cut approach.

Although the global optimization methods have obtained good results for many images, unnatural images are still generated due to two factors: (1) available samples in the data region are quite limited, and (2) pattern similarity is one of the required conditions but is not sufficient for reproducing natural textures. Thus, in order to improve the image quality, these two factors should be considered. There have already been some attempts at this. For (1), the scale and orientation of textures have been considered to obtain effective samples 22 . For (2), Sun et al. 23. and Jia et al. 24 have proposed techniques that use explicit constraints for texture boundaries. These methods synthesize textures preserving the edges or boundaries of the texture. However, automatic and effective determination of these explicit constraints is still difficult.

In this paper, we propose a new approach that is different from conventional ones. For (1), brightness change of sample textures that has not been considered in the literature is allowed to obtain effective samples. For (2), the spatial locality of texture pattern is considered as an implicit constraint that is usually satisfied in a lot of real scenes. In this study, these ideas are implemented with the framework of energy minimization. The effectiveness of our method is demonstrated with subjective and objective evaluation. In addition, in this paper, the validity of evaluation methods for image inpainting which has not been well discussed is analyzed. Furthermore, the problems of qualitative and quantitative evaluation methods that have been used in the literature are clearly demonstrated.

\section{Image Inpainting Based on Energy Minimization}

In the proposed method, after initial values are given to missing regions, the target regions are completed by minimizing an energy function. In the following sections, the definition of an energy function and the minimization method of the energy function are described.

\subsection{Energy Function Considering Brightness Change and Spatial Locality}

As illustrated in Figure1, an image is divided into region $\Omega^{\prime}$ including the missing region $\Omega$ and the data region $\Phi$, which is the rest of the image. The plausibility 


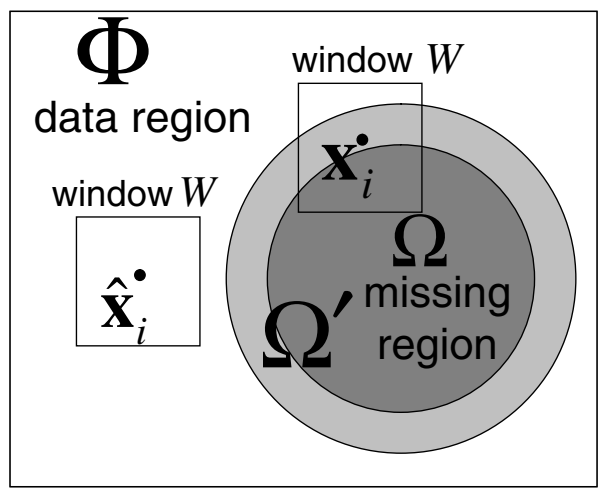

Fig. 1. Data and missing regions in an image

in the missing region $\Omega$ is defined by using image patterns in the data region $\Phi$. Here, $\Omega^{\prime}$ is the expanded area of the missing region $\Omega$. $\Omega^{\prime}$ has a central pixel $\mathbf{x}_{i}$ of a square window $W$ overlapping the region $\Omega$. The energy function is defined as the weighed sum of $S S D^{\prime}$ representing the pattern similarity considering brightness change and $S D$ representing the spatial locality as follows:

$$
E=\sum_{\mathbf{x}_{i} \in \Omega^{\prime}} w_{\mathbf{x}_{i}}\left\{S S D^{\prime}\left(\mathbf{x}_{i}, \hat{\mathbf{x}}_{i}\right)+w_{d i s} S D\left(\mathbf{x}_{i}, \hat{\mathbf{x}}_{i}\right)\right\},
$$

where $\mathbf{x}_{i}$ is the pixel in the region $\Omega^{\prime}, \hat{\mathbf{x}}_{i}$ is the corresponding pixel in the data region $\Phi$ and $w_{d i s}$ is the weight for the spatial locality. Here, $w_{\mathbf{x}_{i}}$ is the weight for pixel $\mathbf{x}_{i}$ and is set as 1 if $\mathbf{x}_{i}$ is inside of the region $\Omega^{\prime} \cap \bar{\Omega}$ because pixel values in this region are fixed: otherwise $w_{\mathbf{x}_{i}}$ is set as $c^{-d}$ ( $d$ is the distance from the boundary of $\Omega$ and $c$ is a constant) because pixel values around the boundary have higher confidence than those in the center of the missing region. In the following, definitions of $S S D^{\prime}\left(\mathbf{x}_{i}, \hat{\mathbf{x}}_{i}\right)$ and $S D\left(\mathbf{x}_{i}, \hat{\mathbf{x}}_{i}\right)$ are described.

\section{Similarity considering brightness change}

The similarity measure $S S D^{\prime}$ is defined as:

$$
S S D^{\prime}\left(\mathbf{x}_{i}, \hat{\mathbf{x}}_{i}\right)=\sum_{\mathbf{p} \in W}\left\{I\left(\mathbf{x}_{i}+\mathbf{p}\right)-\alpha_{\mathbf{x}_{i} \hat{\mathbf{x}}_{i}} I\left(\hat{\mathbf{x}}_{i}+\mathbf{p}\right)\right\}^{2},
$$

where $I(\mathbf{x})$ represents the intensity of pixel $\mathbf{x} . \alpha_{\mathbf{x}_{i} \hat{\mathbf{x}}_{i}}$ is the intensity modification coefficient. By using this coefficient, the brightness of the texture in the data region is adjusted to that in the missing region so as to prevent unnatural brightness changes. In this paper, we employ the ratio of average pixel values around the pixels $\mathbf{x}_{i}$ and $\hat{\mathbf{x}}_{i}$ as the coefficient $\alpha_{\mathbf{x}_{i} \hat{\mathbf{x}}_{i}}$.

$$
\alpha_{\mathbf{x}_{i} \hat{\mathbf{x}}_{i}}=\frac{\sqrt{\sum_{\mathbf{q} \in W} I\left(\mathbf{x}_{i}+\mathbf{q}\right)^{2}}}{\sqrt{\sum_{\mathbf{q} \in W} I\left(\hat{\mathbf{x}}_{i}+\mathbf{q}\right)^{2}}} .
$$




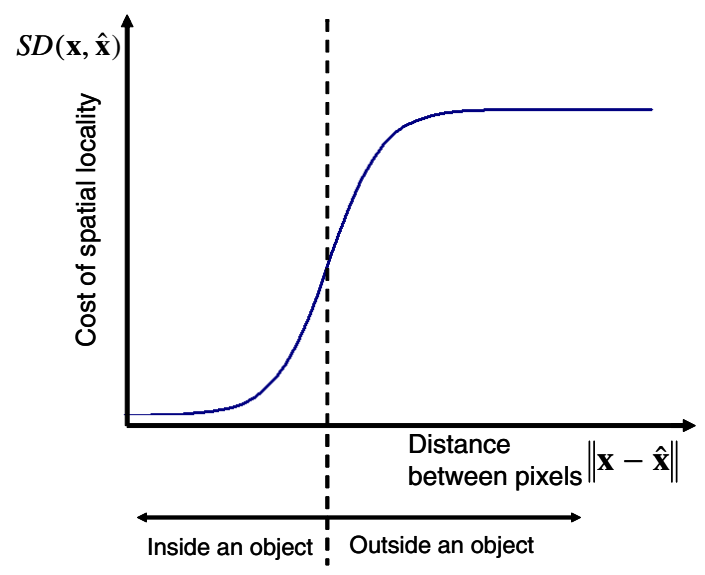

Fig. 2. Sigmoid function for cost of spatial locality

However, an unnatural image is easily generated if large brightness change is approximated by linear transformation. Therefore, we limit the range of the value $\alpha_{\mathbf{x}_{i} \hat{\mathbf{x}}_{i}}$.

\section{Spatial locality of texture}

Cost term $S D$ for spatial locality of a texture pattern is defined by using a sigmoid function:

$$
S D\left(\mathbf{x}_{i}, \hat{\mathbf{x}}_{i}\right)=\frac{\|W\|}{1+e^{\left\{-K\left(\left\|\mathbf{x}_{i}-\hat{\mathbf{x}}_{i}\right\|-X_{0}\right)\right\}}},
$$

where $K$ and $X_{0}$ are constant and $\|W\|$ is the number of pixels in a window. As illustrated in Fiture 2, this cost term is based on the assumption that the probability of similar texture existence for a certain pixel is uniformly high (cost is uniformly low) in the object region which the pixel belongs to. On the other hand, outside the object region, the probability can be assumed to be uniformly low (cost is uniformly high). By adding the constraint of spatial locality, even when the deformation of texture pattern exists around the missing region, appropriate textures that exist near the missing region are preferentially selected.

\subsection{Energy Minimization}

The energy function $E$ in Eq. (1) is minimized by using a framework of greedy algorithm. In our definition of the energy $E$, the energy for each pixel can be treated independently if pattern pairs $\left(\mathbf{x}_{i}, \hat{\mathbf{x}}_{i}\right)$ can be fixed and the change of the coefficient $\alpha_{\mathbf{x}_{i} \hat{\mathbf{x}}_{i}}$ in iteration is very small. Thus, we repeat the following two processes until the energy converges: (i) update of pairs of windows keeping pixel values fixed, and (ii) parallel update of all the pixel values keeping pairs of windows fixed. 
In process (i), the update of the pair of windows is performed by calculating $\hat{\mathbf{x}}_{i}$ keeping the pixel values $I\left(\mathbf{x}_{i}\right)$ fixed. $\hat{\mathbf{x}}_{i}$ is determined as follows:

$$
\hat{\mathbf{x}}_{i}=f\left(\mathbf{x}_{i}\right)=\underset{\mathbf{x} \in \Phi}{\operatorname{argmin}}\left(S S D^{\prime}\left(\mathbf{x}_{i}, \mathbf{x}\right)+w_{\text {dis }} S D\left(\mathbf{x}_{i}, \mathbf{x}\right)\right) .
$$

In process (ii), all the pixel values $I\left(\mathbf{x}_{i}\right)$ are updated in parallel so as to minimize the energy keeping the similar pairs of windows fixed. In the following, the method for calculating the pixel values $I\left(\mathbf{x}_{i}\right)$ is described. First, the energy $E$ is resolved into the element energy $E\left(\mathbf{x}_{i}\right)$ for each pixel in the missing region.

As shown in Figure 3, the target pixel to be updated is $\mathbf{x}_{i}$, and the pixel position inside a window can be expressed as $\mathbf{x}_{i}+\mathbf{p}(\mathbf{p} \in W)$ and is corresponded to $f\left(\mathbf{x}_{i}+\mathbf{p}\right)$ by Eq. (5). Thus, the position of the pixel corresponding to the pixel $\mathbf{x}_{i}$ is $f\left(\mathbf{x}_{i}+\mathbf{p}\right)-\mathbf{p}$. Now, the element energy $E\left(\mathbf{x}_{i}\right)$ can be expressed in terms of the pixel values of $\mathbf{x}_{i}$ and $f\left(\mathbf{x}_{i}+\mathbf{p}\right)-\mathbf{p}$, the coefficient $\alpha$ and the Euclid distance between $\mathbf{x}_{i}$ and $f\left(\mathbf{x}_{i}\right)$ as follows:

$$
\begin{aligned}
E\left(\mathbf{x}_{i}\right)=\sum_{\mathbf{p} \in W} w_{\left(\mathbf{x}_{i}+\mathbf{p}\right)}\left\{I\left(\mathbf{x}_{i}\right)-\alpha_{\left(\mathbf{x}_{i}+\mathbf{p}\right) f\left(\mathbf{x}_{i}+\mathbf{p}\right)} I\left(f\left(\mathbf{x}_{i}+\mathbf{p}\right)-\mathbf{p}\right)\right\}^{2} \\
\\
+w_{d i s} \frac{\|W\|}{1+e^{\left\{-K\left(\left\|\mathbf{x}_{i}-f\left(\mathbf{x}_{i}\right)\right\|-X_{0}\right)\right\}}} .
\end{aligned}
$$

The relationship between the energy $E$ for all of the missing region and the element energy $E\left(\mathbf{x}_{i}\right)$ for each pixel can be written as follows:

$$
E=\sum_{\mathbf{x}_{i} \in \Omega} E\left(\mathbf{x}_{i}\right)+C .
$$

$C$ is the energy of pixels in the region $\bar{\Omega} \cap \Omega^{\prime}$, and is treated as a constant because pixel intensities in the region and all pairs of windows are fixed here. Therefore, by minimizing the element energy $E(\mathbf{x})$ respectively, the total energy $E$ can be minimized. Here, if it is assumed that the change of $\alpha_{\mathbf{x}_{i} f\left(\mathbf{x}_{i}\right)}$ is smaller than that of the pixel intensity $I\left(\mathbf{x}_{i}\right)$, by differentiating $E\left(\mathbf{x}_{i}\right)$ with respect to $I\left(\mathbf{x}_{i}\right)$, each pixel value $I\left(\mathbf{x}_{i}\right)$ in the missing region can be calculated in parallel as follows:

$$
I\left(\mathbf{x}_{i}\right)=\frac{\sum_{\mathbf{p} \in W} w_{\left(\mathbf{x}_{i}+\mathbf{p}\right)} \alpha_{\left(\mathbf{x}_{i}+\mathbf{p}\right) f\left(\mathbf{x}_{i}+\mathbf{p}\right)} I\left(f\left(\mathbf{x}_{i}+\mathbf{p}\right)-\mathbf{p}\right)}{\sum_{\mathbf{p} \in W} w_{\left(\mathbf{x}_{i}+\mathbf{p}\right)}} .
$$

Additionally, in order to avoid local minima efficiently, a coarse-to-fine approach is also employed for energy minimization. Specifically, an image pyramid is generated and the energy minimization processes (i) and (ii) are repeated from higher-level to lower-level layers successively using a certain size of window. Good initial values are given to the lower layer by projecting results from the higher layer. This makes it possible to decrease computational cost and avoid local minima. In the lowest layer (original size), the energy minimization process is repeated while reducing the size of the window, and it enables reproduction of more detailed textures. 


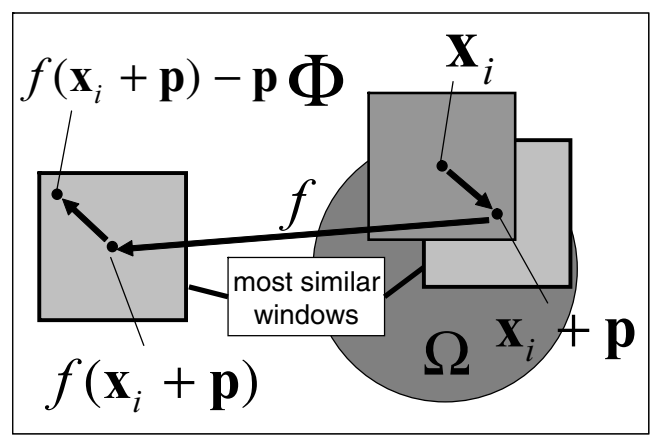

Fig. 3. Relationship between pixels in energy calculation

\section{Experiments}

In experiments, we have compared the proposed method with conventional ones using 100 images. The methods are our implemented Criminisi's method (Method A) [17, our implemented Wexler's method (Method B) 20] and the proposed method (Method E). To confirm the effectiveness of considering the brightness change and spatial locality, respectively, the proposed method allowing only brightness change (Method $\mathrm{C}$ ) and the proposed method considering only spatial locality (Method D) were also evaluated. In these experiments, each parameter in the energy function was set as shown in Table 1 for all the images. Here, the missing region was manually specified, and the average pixel value of the boundary of the missing region was given as an initial value in the missing region.

\subsection{Qualitative Evaluation}

All the completed images by 5 methods were subjectively evaluated by 37 subjects. The subjects were requested to access the web page for questionnaire evaluation and evaluate the resultant images arranged in random order by giving a score of 1 (bad) to 5 (good).

Table 1. Parameters in experiments

\begin{tabular}{|cc|c|}
\hline Window size & $N_{w}$ & $\begin{array}{c}\max 9 \times 9 \\
\min 3 \times 3\end{array}$ \\
\hline Weight for distance & $w_{d i s}$ & 120 \\
\hline Parameter in sigmoid function & $K$ & 0.4 \\
& $X_{0}$ & 20 \\
\hline Range of coefficient $\alpha$ & $D$ & 0.1 \\
\hline
\end{tabular}


The average scores of the 100 resultant images are shown in Table 2 for each method 1 Table 2 shows that the average score by the proposed method (method $\mathrm{E}$ ) is higher than the scores by the conventional methods (methods A and B). In this experiment, scores of the proposed method (method E) and the conventional methods (methods A and B) were compared by using the t-test with a $5 \%$ significant level. Resultingly, significant difference was observed between these scores. In addition, both methods $\mathrm{C}$ and $\mathrm{D}$ obtained higher scores than methods $\mathrm{A}$ and $\mathrm{B}$ and significant differences were also observed by using the t-test with a $5 \%$ significant level. Therefore, the proposed method can be statistically verified to be better than the conventional methods $\mathrm{A}$ and $\mathrm{B}$, and considering brightness change and spatial locality is respectively effective. Figure 4 shows example images for which the proposed method had better scores. The proposed method has generated better results for the images in which brightness change and deformation of texture patterns exist around the missing regions due to the illumination change and the perspective projection effect.

\subsection{Quantitative Evaluation}

In this experiment, RMSE was computed for 29 images whose missing regions were specified regardless of the object regions, and we did not use the remaining 71 images because the missing regions of the 71 images are specified so as to remove certain objects. Table 3 shows the average RMSE. From the results, the proposed method is the best of all 5 the methods and significant differences between the proposed (E) and conventional methods (A and B) were observed by using the t-test with a $5 \%$ significant level.

\subsection{Discussion of Evaluation Methods}

Although some evaluation have been done in the literature of image inpainting, the validity of evaluation methods has not been well discussed. In this section, qualitative and quantitative evaluation methods are analyzed using our results and their problems are clarified.

Table 2. Average score from 100 images

\begin{tabular}{|c|c|c|c|c|c|}
\hline Method & A & B & C & D & E \\
\hline Average score & 2.21 & 3.24 & 3.39 & 3.42 & 3.60 \\
\hline
\end{tabular}

Table 3. Average RMSE from 29 images

\begin{tabular}{|c|c|c|c|c|c|}
\hline Method & A & B & C & D & E \\
\hline RMSE & 42.95 & 28.40 & 27.83 & 28.36 & 27.44 \\
\hline
\end{tabular}
${ }^{1}$ One hundred input and resultant images are shown on the web page
[http://yokoya.naist.jp/research/inpainting/]. 

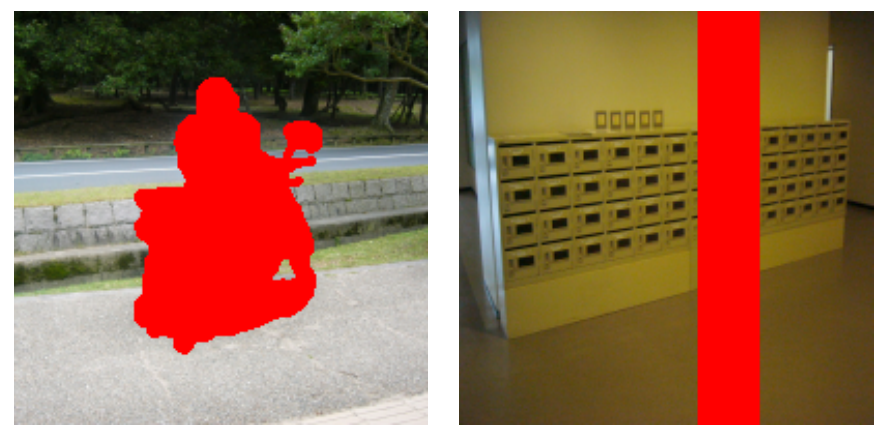

(a) Input images with a missing region.
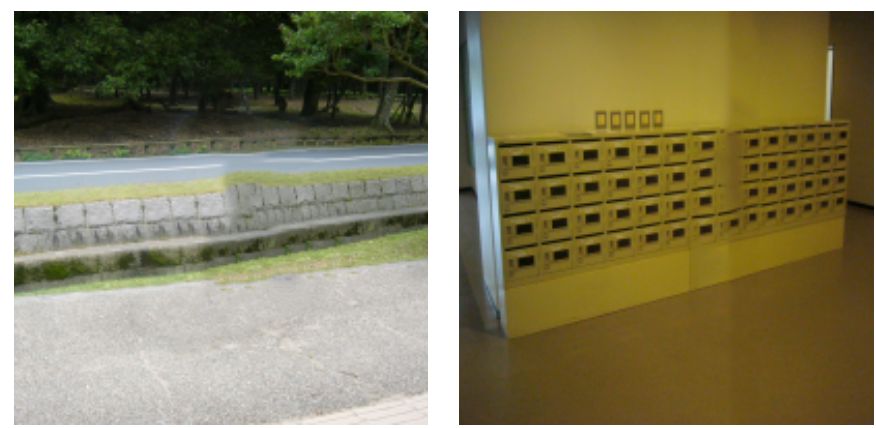

(b) Results by Wexler's method [20].
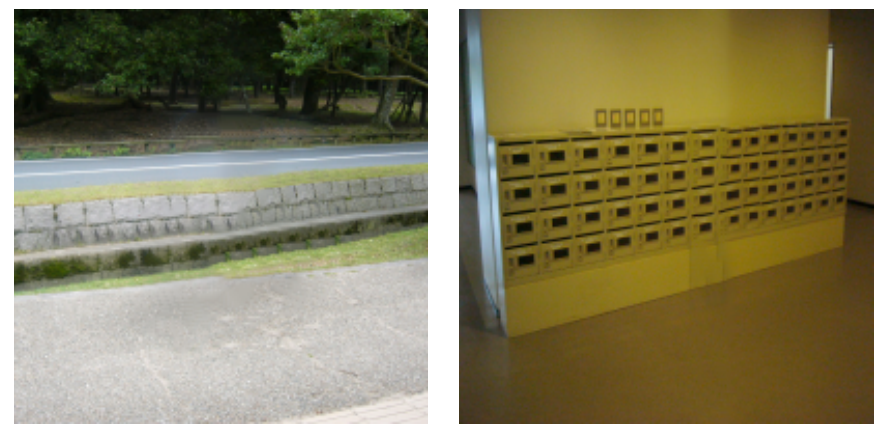

(c) Results by the proposed method.

Fig. 4. Example images providing the proposed method with better scores

\section{Reliability of qualitative evaluation}

The evaluation using a few images and a few subjects may not be able to validate the effectiveness of inpainting methods due to the bias of images and subjects. In this section, the reliability of the result when the images and subjects are 


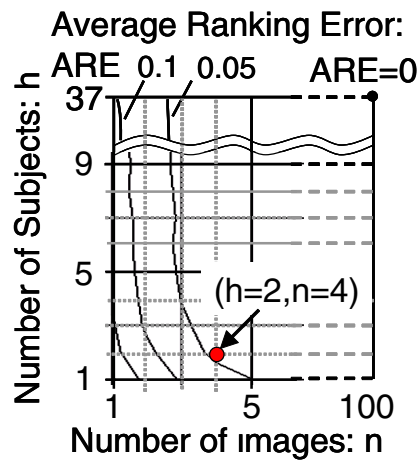

(a) For A and E.

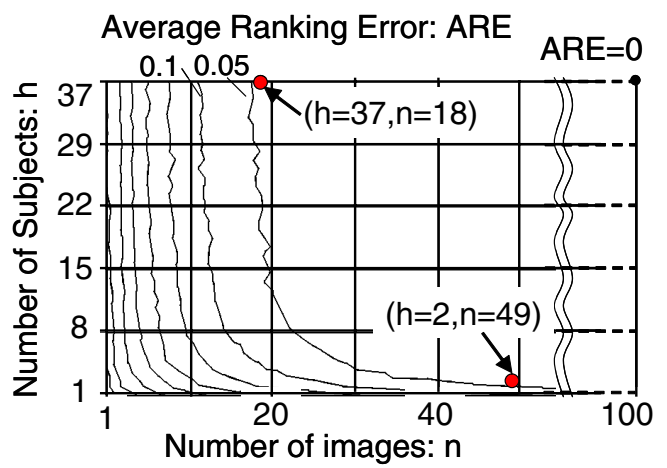

(b) For D and E.

Fig. 5. Relationships among ARE and the number of images and subjects

decreased in a questionnaire is analyzed in simulation. Here, the ranking of the methods decided by the average of scores given by 37 subjects in the questionnaire using 100 images were used as the ground truth.

In this paper, as the reliability measure of evaluation results, RE (Ranking Error) is defined as:

$$
R E(n, h)= \begin{cases}0 & (\forall i, g(i, 100,37)-g(i, n, h)=0) \\ 1 & (\text { otherwise })\end{cases}
$$

where $g(i, n, h)$ represents the rank order of the method $i$ determined by $n$ images and $h$ subjects. In this study, we compute $R E(n, h)$ with respect to random selection of $n$ images and $h$ subjects from 100 images and 37 subjects. $A R E(n, h)$ is the average score of $R E(n, h)$ by 10,000 times selection.

Figure [5] shows the relationship among ARE and the number of images and subjects. In this figure, (a) and (b) illustrate isolines of ARE for the methods A and $\mathrm{E}$, and $\mathrm{D}$ and $\mathrm{E}$, respectively. In this figure, isolines are drawn every 0.05.

The scores of the methods $\mathrm{A}$ and $\mathrm{E}$ are significantly and largely different. From Figure 5(a), when $h=2$, the ranking corresponds to the ground truth more than $95 \%$ of the time $(A R E=0.05)$ if $n>3$. Therefore, for methods that generate significantly different results, the ranking is usually equivalent to the ground truth even if the numbers of subjects and images are small. On the other hand, the scores of the methods D and E are significantly but a little different. From Figure 5(b), in order for the ranking to correspond to the ground truth more than $95 \%$ of the time, more than 49 images are needed if $h=2$. Even if $h=37$, at least 18 images are needed. Therefore, if the numbers of subjects and images are small, the ranking may not correspond to the ground truth and the result of subjective evaluation is not reliable. Therefore, to evaluate image inpainting methods persuasively, many images and subjects (for example, 40 images and 5 subjects) are needed. 


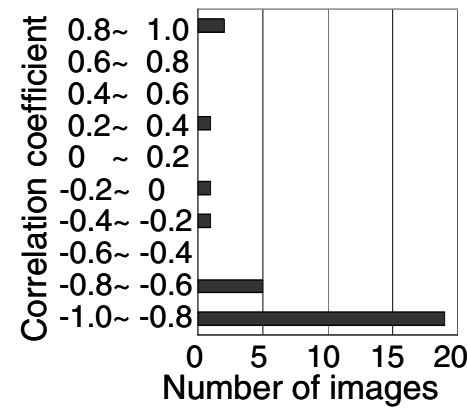

(a) From A to E.

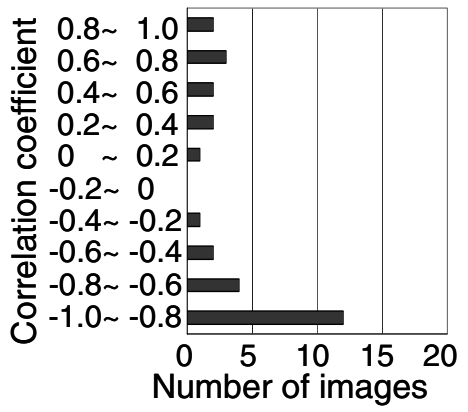

(b) From B to E.

Fig. 6. Correlation coefficient between RMSE and subjective score
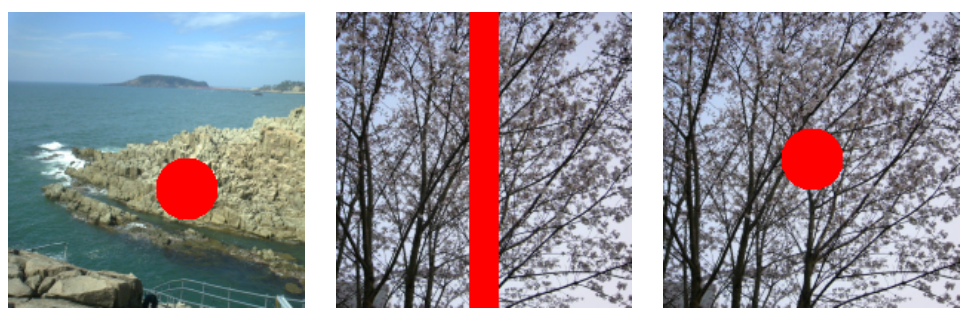

Fig. 7. Images with inverse correlation between RMSE and subjective evaluations

\section{Relationship between quantitative and qualitative evaluation}

In the literature on image inpainting, as the quantitative evaluation, MSE (Mean Squared Error), RMSE (Root Mean Squared Error) and PSNR (Peak Signal-to-Noise Ratio), which are based on computing pixel-wise differences between original and inpainted images, have been often used. In this evaluation, RMSE is computed for each image, and the relationship between RMSE and scores given by subjects is discussed.

Figure [6 illustrates the distribution of the correlation coefficient between RMSE and the subjective score for 29 images: (a) is by 5 methods, (b) is by 4 methods except for method A [17. Correlation coefficient $C c$ is computed as follows:

$$
C c=\frac{N_{m} \Sigma_{i}^{N_{m}} R_{i} S_{i}-\Sigma_{i}^{N_{m}} R_{i} \Sigma_{i}^{N_{m}} S_{i}}{\sqrt{N_{m} \Sigma_{i}^{N_{m}} R_{i}{ }^{2}-\left(\Sigma_{i}^{N_{m}} R_{i}\right)^{2}} \sqrt{N_{m} \Sigma_{i}^{N_{m}} S_{i}{ }^{2}-\left(\Sigma_{i}^{N_{m}} S_{i}\right)^{2}}},
$$

where $N_{m}$ is the number of methods, $R_{i}$ is RMSE and $S_{i}$ is the average score by subjects. From Table 3 and Figure 6(a), there is a clear negative correlation between RMSE and subjective score. From this, although the evaluation result by RMSE may correspond to the ground truth, there also exist three images (Figure 7) where positive correlation exists. As shown in Figure 7 , these images 
have very high-frequency components. It is well known that pixel correlationbased similarity measures including RMSE are sensitive to pixel phase shift for a high frequency component. However, such a pixel phase shift does not always affect the naturality of images. Thus, RMSE cannot appropriately evaluate the images with a high frequency component. Figure 6(b) illustrates the distribution of the correlation coefficient by the 4 methods except for method A, whose score is extremely low. In Figure 6(b), one third of the coefficients indicate positive correlation. This means that the small difference in RMSE does not always represent that of image naturality. Therefore, RMSE cannot be used as an absolute criterion of image naturality but can be used for a rough evaluation.

\section{Conclusion}

In this paper, the new energy function for image inpainting has been proposed. To obtain good results for many images, two factors were considered: (1) brightness change of sample textures was allowed, (2) spatial locality was introduced as a new constraint. By considering these two factors, the missing region was completed successfully for many images. In experiments, we have demonstrated the effectiveness of our method by qualitative and quantitative evaluation. we have also discussed the validity of evaluation methods for image inpainting. In future work, we should establish a method to decide optimum parameters automatically by analyzing an image.

Acknowledgments. This research was partially supported by the Ministry of Education, Culture, Sports, Science and Technology, Grant-in-Aid for Scientific Research (A), 19200016.

\section{References}

1. Levin, A., Zomet, A., Weiss, Y.: Learning How to Inpaint from Global Image Statistics. In: Proc. ICCV, vol. 1, pp. 305-312 (2003)

2. Ballester, C., Bertalmio, M., Sapiro, V., Verdera, J.: Filling-In by Joint Interpolation of Vector Fields and Gray Levels. Trans. on Image Processing 10(8), 1200-1211 (2001)

3. Ballester, C., Caselles, V., Verdera, J., Bertalmio, M., Sapiro, G.: A Variational Model for Filling-In Gray Level and Color Images. In: Proc. ICCV, pp. 10-16 (2001)

4. Tschumperlé, D.: Curvature-preserving regularization of multi-valued images using pDE's. In: Leonardis, A., Bischof, H., Pinz, A. (eds.) ECCV 2006. LNCS, vol. 3952, pp. 295-307. Springer, Heidelberg (2006)

5. Villéger, E., Aubert, G., Blanc-Féraud, L.: Image Disocclusion Using a Probabilistic Gradient Orientation. In: Proc. ICPR, vol. 2, pp. 52-55 (2004)

6. Bertalmio, M., Bertozzi, A.L., Sapiro, G.: Navier-Stokes, Fluid Dynamics, and Image and Video Inpainting. In: Proc. CVPR, pp. 355-362 (2001) 
7. Bertalmio, M., Sapiro, G., Caselles, V., Ballester, C.: Image Inpainting. In: Proc. SIGGRAPH 2000, pp. 417-424 (2000)

8. Esedoglu, S., Shen, J.: Digital Inpainting Based on the Mumford-shah-euler Image Model. European J. of Applied Mathematics 13, 353-370 (2003)

9. Masnou, S., Morel, J.M.: Level Lines Based Disocclusion. In: Proc. ICIP, vol. 3, pp. 259-263 (1998)

10. Chan, T., Shen, J.: Non-texture Inpainting by Curvature-Driven Diffusions (CDD). J. of Visual Communication and Image Representation 12(4), 436-449 (2001)

11. Chan, T., Kang, S., Shen, J., Osher, S.: Euler's Elastica and Curvature Based Inpaintings. SIAM J. of Applied Mathematics 63(2), 564-592 (2002)

12. Hirani, A.N., Totsuka, T.: Combining Frequency and Spatial Domain Information for Fast Interactive Image Noise Removal. In: Proc. SIGGRAPH 1996, pp. 269-276 (1996)

13. Rane, S.D., Remus, J., Sapiro, G.: Wavelet-Domain Reconstruction of Lost Blocks in Wireless Image Transmission and Packet-Switched. In: Proc. ICIP, vol. 1, pp. 309-312 (2002)

14. Amano, T.: Image Interpolation by High Dimensional Projection Based on Subspace Method. In: Proc. ICPR, vol. 4, pp. 665-668 (2004)

15. Efros, A.A., Leung, T.K.: Texture Synthesis by Non-parametric Sampling. In: Proc. ICCV, pp. 1033-1038 (1999)

16. Bornard, R., Lecan, E., Laborelli, L., Chenot, J.: Missing Data Correction in Still Images and Image Sequences. In: Proc. ACM Int. Conf. on Multimedia, pp. 355-361 (2002)

17. Criminisi, A., Pérez, P., Toyama, K.: Region Filling and Object Removal by Exemplar-Based Image Inpainting. Trans. on Image Processing 13(9), 1200-1212 (2004)

18. Li, B., Qi, Y., Shen, X.: An Image Inpainting Method. In: Proc. IEEE Int. Conf. on Computer Aided Design and Computer Graphics, pp. 531-536 (2005)

19. Allène, C., Paragios, N.: Image Renaissance Using Discrete Optimization. In: Proc. ICPR, pp. 631-634 (2006)

20. Wexler, Y., Shechtman, E., Irani, M.: Space-Time Completion of Video. Trans. on PAMI 29(3), 463-476 (2007)

21. Komodakis, N., Tziritas, G.: Image Completion Using Global Optimization. In: Proc. CVPR, pp. 442-452 (2006)

22. Drori, I., Cohen-Or, D., Yeshurun, H.: Fragment-Based Image Completion. In: Proc. SIGGRAPH 2003, pp. 303-312 (2003)

23. Sun, J., Yuan, L., Jia, J., Shum, H.: Image Completion with Structure Propagation. In: Proc. SIGGRAPH 2005, pp. 861-868 (2005)

24. Jia, J., Tang, C.: Image Repairing: Robust Image Synthesis by Adaptive ND Tensor Voting. In: Proc. CVPR, pp. 643-650 (2003) 